\title{
Psychometric assessment of the Brazilian version of the Male Body Dissatisfaction Scale
}

\author{
Pedro Henrique Berbert de Carvalho ${ }^{1}$, Maria Aparecida Conti2, Clara Mockdece Neves³, Juliana Fernandes \\ Filgueiras Meireles 3 , Fernanda Costa Oliveira ${ }^{3}$, Maria Elisa Caputo Ferreira ${ }^{3}$
}

1 Universidade Federal de Juiz de Fora (UFJF) - Campus Governador Valadares.

2 Universidade Cruzeiro do Sul.

3 UFJF-Campus Juiz de Fora.

Received: 2/10/2015 - Accepted: 7/28/2015

DOI: 10.1590/0101-60830000000056

\begin{abstract}
Background: The study of male body image has increased substantially, but there are few assessment tools available for this population. The Male Body Dissatisfaction Scale (MBDS) has been widely used among students to research body image disturbances and eating disorders. However, the psychometric properties of this instrument have not been tested in the Brazilian context. Objectives: To explore the psychometric properties (convergent validity, internal consistency, test-retest reliability and factor structure) of the Brazilian version of the MBDS. Methods: Two-hundred sixty-four undergraduate students were evaluated. Pearson's correlation was used to test the convergent validity of the MBDS and the Drive for Muscularity Scale, the Swansea Muscularity Attitudes Questionnaire, the Rosenberg Self-Esteem Scale, the Beck Depression Inventory, the Eating Attitudes Test-26, and the Commitment to Exercise Scale. Test-retest reliability was evaluated using t-tests for repeated measures and by calculating the coefficient of intraclass correlation. Exploratory factor analysis was conducted, and Cronbach's a coefficients were determined. A significance level of $5 \%$ was adopted. Results: The MBDS had an adequate factor structure, with two factors explaining $52.67 \%$ of the total variance. It showed excellent internal consistency (Cronbach's $\alpha$ between 0.90 and 0.92 ), a high intraclass correlation coefficient (0.81), and convergent validity with the drive for muscularity, the psychological commitment to exercise, low self-esteem, and eating disorder risk behaviour measures. Discussion: The MBDS appears to be a valid and reliable tool for evaluating Brazilian male body image dissatisfaction.
\end{abstract}

Carvalho PHB et al. / Arch Clin Psychiatry. 2015;42(4):90-4

Keywords: Body image, men’s health, psychometrics, validation, factor analysis.

\section{Introduction}

Body image dissatisfaction, a negative feeling about one's appearance ${ }^{1}$ that has been previously associated with women ${ }^{2}$, is currently being investigated in males in many countries ${ }^{3-5}$. In both women and men, body dissatisfaction is associated with low self-esteem, depressive symptoms and risk behaviours associated with eating disorders ${ }^{6}$.

The concern with physical appearance and body image is a reality in many developed and developing countries and is closely related to health and illness 7 . In Brazil, the body is a social status symbol, and physical appearance is an essential element in the construction of a national Brazilian identity. The country was ranked first worldwide in the total number of plastic surgery procedures ${ }^{9}$, and it stands out for the number of surgical liposuction, blepharoplasty and rhinoplasty procedures performed, demonstrating the importance of physical appearance for Brazilians.

A population-based study conducted in Brazil 5 found a $60.5 \%$ prevalence rate for body dissatisfaction among men. The study pointed to an association between weight dissatisfaction and physical inactivity, alcohol abuse, obesity and old age. The authors also found an association between dissatisfaction with muscularity and low body mass index and common mental disorders. Santos Silva et al. ${ }^{5}$ highlighted the need to assess body image dissatisfaction because of its influence on the individual's health and quality of life.

There are some concerns specific to the assessment of male body dissatisfaction. Cafri and Thompson ${ }^{10}$ highlighted the need to: a) assess satisfaction with muscularity, b) identify behaviours associated with body dissatisfaction, and c) evaluate satisfaction with specific body parts/regions, such as the shoulders, chest and arms. The authors ${ }^{10}$ emphasised that a concern with muscularity is the central aspect of male body image. It should also be noted that dissatisfaction with muscularity is a concern among individuals with muscle dysmorphia, a very specific type of body dysmorphic disorder characterised by a fear of being too small and by perceiving oneself as small and weak even when one is actually large and muscular ${ }^{3,6}$.

Several authors $2,10-12$ who were aware of these methodological concerns have contributed to psychometric assessment and/or discussions in the field of male body image assessment. Tod et al.12 highlighted four instruments with good psychometric properties: the Drive for Muscularity Scale ${ }^{13}$, the Drive for Muscularity Attitudes Questionnaire ${ }^{14}$, Yelland and Tiggemann's ${ }^{15}$ Drive for Muscularity Scale, and the Swansea Muscularity Attitudes Questionnaire ${ }^{16}$.

Two of these instruments have been analysed psychometrically for use with Brazilian males: the Drive for Muscularity Scale and the Swansea Muscularity Attitudes Questionnaire ${ }^{17}$. However, it should be noted that both instruments were developed to assess the drive for muscularity, which is related to dissatisfaction with body muscularity but is a different construct. In a systematic review, Carvalho and Ferreira ${ }^{2}$ pointed to the growth in the number of adapted and/or validated instruments for young Brazilian adults. However, despite the growing number of scales and questionnaires for evaluating male body image, none of these instruments allows the respondent to evaluate the relative importance of each item, which can contribute to overestimating or underestimating the value of an item in the total score.

Based on this premise and given the lack of instruments that specifically evaluate the construct of dissatisfaction with body muscularity, Ochner et al. ${ }^{18}$ developed the Male Body Dissatisfaction Scale (MBDS). Initial evidence of the validity of the MBDS 18 included its good psychometric properties, with excellent internal consistency (Cronbach's $\alpha=0.93$ ) and its convergent validity with self-esteem (Rosenberg Self-Esteem Scale; $r=-0.33, p<0.05$ ), body self-esteem (Shape and Weight-Based Self-Esteem Scale; $r=0.53$, $\mathrm{p}<0.01$; and Body Esteem Scale; $\mathrm{r}=-0.29, \mathrm{p}<0.05)$; and self-reported body satisfaction measures ( 0 to 100 points, $r=-0.52, p<0.01$ ). In addition, the scale showed good temporal stability (test-retest) of 
0.95 ( $\mathrm{p}<0.0005)$. Through exploratory factor analysis, Ochner et al. ${ }^{18}$ proposed a factorial structure with three factors: (1) musculature (items 4, 6, 7, 9, 12, 13, 16 and 24), (2) definition (items 1, 3, 10, 15, $17,18,20,22$ and 25) and (3) relative standing/external evaluation (items 2, 5, 8, 11, 14, 19, 21 and 23).

The MBDS was previously evaluated for use with young Brazilian adults (18-30 years old) through an analysis of its conceptual, semantic and instrumental equivalence and an analysis of its internal consistency ${ }^{19}$. Translation, back-translation, translation synthesis, expert committee evaluation, pre-test, and internal consistency analysis were performed. The MBDS was translated into and adapted to the Portuguese language while maintaining its 25 original items and was shown to be easily understandable with adequate internal consistency $(\alpha=0.92)^{19}$.

Although the instrument showed good initial validity, other indicators must also be evaluated. Therefore, the purpose of this study was to explore the psychometric properties (convergent validity, internal consistency, test-retest reliability, and factor structure) of the Brazilian version of the MBDS ${ }^{19}$. It is believed that such an instrument may be useful as an evaluation tool for clinical and epidemiological research.

\section{Method}

\section{Participants and procedures}

The sample was composed of 264 Brazilian men with a mean age of 20.13 years (standard deviation $[\mathrm{SD}]=1.71$ years; range $18-30$ years) and a mean body mass index (BMI) of $23.12 \mathrm{~kg} / \mathrm{m}^{2}\left(\mathrm{SD}=3.07 \mathrm{~kg} / \mathrm{m}^{2}\right.$; range $16.78-41.22 \mathrm{~kg} / \mathrm{m}^{2}$ ). BMI was calculated using self-reported weight and height. The participants were from diverse academic departments (Computer Science, Law, Civil Engineering, Electrical Engineering, Philosophy, Physiotherapy, Mathematics, Medicine and Psychology) at the Federal University of Juiz de Fora, Minas Gerais, Brazil and were selected by convenience based on their presence in the classroom and willingness to complete the questionnaires.

After the researchers contacted the course coordinators and teachers, the subjects were addressed in groups during class. They received information about the research objectives and the procedures to be followed, and the anonymity of each participant was ensured. All of the participants signed a written informed consent form authorising their voluntary participation. Then, the participants answered the questionnaires individually. The study was approved by the Research Ethical Board of Clinical Hospital, Medical School, University of São Paulo (protocol number - 0586/08).

\section{Instruments}

The Brazilian version of the MBDS ${ }^{19}$ contains 25 items rated on a 5 -point scale ranging from 1 (always/strongly agree) to 5 (never/ strongly disagree); 13 items were reverse-scored (items 4, 5, 6, 7, 9, $10,12,13,16,17,22,24$ and 25). In addition to the evaluation along the 5-point Likert scale, the participants rated the importance of each item to them on a scale of 1 to 10 . To calculate the total score, the value of each item (degree of importance) was divided by 10 and then multiplied by the Likert scale response to the item (1 to 5). The total MBDS score can range from 2.5 to 125, with higher scores indicating greater body dissatisfaction ${ }^{18}$.

The Drive for Muscularity Scale (DMS) ${ }^{13}$ was used to assess the drive for muscularity. The DMS is a self-report scale consisting of 15 items answered using a 6-point Likert-type response format $(1=$ always to $6=$ never). Total scores range from 15 to 90 points. Higher scores represent more troubling attitudes and behaviours toward muscularity. The scale was translated and adapted for young Brazilian adults and had good psychometric properties ${ }^{17}$. The DMS was used to test the convergent validity of the MBDS.

The Swansea Muscularity Attitudes Questionnaire (SMAQ) ${ }^{16,17}$, another measure that assesses the drive for muscularity, was used to test the convergent validity of the MBDS. The SMAQ ${ }^{17}$ consists of 20 items answered using a 7-point Likert-type response format (definitely, strongly agree, agree, neutral, disagree, strongly disagree and definitely not). The total score ranges from 20 to 140 points. Higher scores indicate a higher drive for muscularity.

The Rosenberg Self-Esteem Scale (RSS)20,21 is a widely used measure with strong reliability and validity ${ }^{21}$. The RSS consists of 10 items rated on a 4-point scale ranging from 1 (strongly agree) to 4 (strongly disagree), with a total score ranging from 10 to 40 points. Higher scores indicate a more positive sense of self. We used the RSS $^{21}$ to test the convergent validity of the MBDS.

To evaluate depressive symptoms, we used the Beck Depression Inventory $(\mathrm{BDI})^{22}$. The instrument consists of 21 items rated on a 4-point scale ranging from 0 to 3 . The total score can range from 0 to 63 points, with higher scores indicating greater degrees of depression. We used the Brazilian version of the $\mathrm{BDI}^{23}$ to test the convergent validity of the MBDS.

The Eating Attitudes Test (EAT-26)24 is a widely used measure of symptoms and troubling characteristics of eating disorders that displays good psychometric properties. The EAT-26 is a self-report instrument comprising 26 items rated with a 3-point Likert scale format $(0=$ never, rarely and very rarely, $1=$ often, $2=$ very often; 3 $=$ always). Item 25 is reverse-scored. According to Nunes et al. 25 , the EAT-26 has good psychometric properties and satisfactory internal consistency. The EAT-26 was used to assess the convergent validity of the MBDS.

Males with high body dissatisfaction generally engage in regular physical exercise (i.e., weightlifting) ${ }^{6}$. To assess this characteristic, we used the Commitment to Exercise Scale (CES) ${ }^{26}$ as a measure of convergent validity. The CES is a visual analogue scale consisting of 8 questions that refer to an individual's commitment to and behaviours and attitudes towards physical exercise. Each question presents a $155-\mathrm{mm}$ line with two response options, one at the beginning and one at the end of the line. The distance between the beginning of the line and the point the respondent marks is the score for each item. Scores range from 0 to 1,240 points, and the higher the score, the higher the respondent's degree of psychological commitment to physical exercise 26 .

\section{Data analysis}

Descriptive analyses (mean, standard deviation, minimum and maximum value) were performed for the scores obtained from the MBDS, DMS, SMAQ, RSS, BDI, EAT-26 and CES. To assess the reliability of the applied instruments, Cronbach's a was estimated for each scale; the results are reported in table 1. Cronbach's a higher than 0.70 were considered adequate ${ }^{27}$.

The Kolmogorov-Smirnov test was used to determine normality in the data distribution. Evidence of convergent validity was evaluated with parametric statistical analyses using Pearson's test of association between the scores of the instruments (DMS, SMAQ, RSS, BDI, EAT26 and CES) and the MBDS. Correlations of $0.20,0.40$, and 0.60 were considered small, moderate, and strong, respectively ${ }^{28}$.

To determine the test-retest reliability of the MBDS, a subset of the sample was randomly selected to re-take the test after two weeks ${ }^{29}$. Fifty-three subjects participated in this phase of the study. They had a mean age of 19.89 years ( $\mathrm{SD}=1.76$ years) and a mean BMI of $22.81 \mathrm{~kg} / \mathrm{m}^{2}\left(\mathrm{SD}=2.94 \mathrm{~kg} / \mathrm{m}^{2}\right)$. The MBDS test-retest reliability was evaluated by comparing the scores obtained (Student's $t$ test for paired measures) between the two points in time and calculating the intraclass correlation coefficient (ICC). In addition, the internal consistency was verified by calculating Cronbach's $\alpha$-coefficient for the two time points ${ }^{29}$.

The MBDS met the standard criteria for multivariate normality with a Mardia coefficient near zero ${ }^{29}$. An exploratory factor analysis (EFA) of the MBDS was then conducted. For the initial MBDS validity analysis, Ochner et al. ${ }^{18}$ included a small sample (fewer than 100 individuals), which some authors 29,30 consider the minimum sample size for the EFA. A ratio of 5 to 20 individuals for each item of the 
instrument, with 10 subjects per item considered an appropriate value $^{29,30}$, is indicated for validity analyses. The MBDS consists of 25 items, which requires a minimum of 250 subjects for the factor analysis. For this reason, the factor structure used in the original study of the MBDS ${ }^{18}$ was not considered as a basis for the EFA of the Brazilian version.

Therefore, an EFA was conducted using principal components analysis, followed by Varimax orthogonal rotation and the use of Kaiser criterion (minimal eigenvalue $=1$ ) for the factor extraction ${ }^{29}$. The data's suitability for this analysis was assessed using the KaiserMeyer-Olkin (KMO) measure of sampling adequacy and Bartlett's test of sphericity. The factor loading matrix was analysed to identify the items and their correspondence with the factors. A cut-off point of 0.40 was used for the item loading values ${ }^{30}$. Cronbach's a was evaluated for each identified factor to determine the internal consistency.

SPSS v.17.0 software was used for all of the analyses, and a significance level of $5 \%$ was adopted.

\section{Results}

\section{Descriptive analysis and convergent validity}

The scores obtained and the correlations between the instruments are summarised in table 1 . It is noteworthy that the instruments showed a significant variation in the scores obtained that comprised almost all possible scoring variations for each instrument (minimum score to maximum score). It can also be noted that all of the instruments had adequate internal consistency ${ }^{27}$.

As expected, the MBDS was associated with almost all of the instruments, indicating convergent validity with various measures (Table 1). Following the cut-offs indicated by Tabachnick and Fidell 28 , the MBDS showed a direct and strong association with the drive for muscularity measures, the DMS and the SMAQ, and the psychological commitment to exercise. A moderate association was also found between the MBDS and eating disorder risk behaviours, and a small and inverse association was found with self-esteem. Only depressive symptoms (BDI) were not associated with the MBDS.

Table 1. Descriptive statistics, Cronbach's $\alpha$ and correlations $(n=264)$

\begin{tabular}{|l|c|c|c|c|c|}
\hline Measure & M & SD & Min-Max & $\begin{array}{c}\text { Cronbach's } \\
\alpha\end{array}$ & $\begin{array}{c}\text { Correlation } \\
\text { with MBDS }\end{array}$ \\
\hline MBDS & 47.76 & 20.19 & $2.90-102.20$ & 0.92 & - \\
\hline DMS & 41.34 & 16.13 & $15-84$ & 0.90 & $0.68^{*}$ \\
\hline SMA0 & 57.23 & 18.37 & $20-100$ & 0.95 & $0.77^{*}$ \\
\hline RSS & 25.41 & 2.95 & $10-35$ & 0.80 & $-0.14^{* *}$ \\
\hline BDI & 6.46 & 4.63 & $0-24$ & 0.76 & 0.08 \\
\hline EAT-26 & 11.29 & 8.45 & $0-51$ & 0.82 & $0.27^{*}$ \\
\hline CES & 52.06 & 28.62 & $0-145.90$ & 0.83 & $0.60^{*}$ \\
\hline
\end{tabular}

M: mean; SD: standard deviation; Min: minimum; Max: maximum; MBDS: Male Body Dissatisfaction Scale; DMS: Drive for Muscularity Scale; SMAQ: Swansea Muscularity Attitudes Questionnaire; RSS: Rosenberg Self-Esteem Scale; BDI: Beck Depression Inventory; EAT-26: Eating Attitudes Test-26; CES: Commitment to Exercise Scale.

${ }^{*} p<0.001$.

${ }^{* *} p<0.05$.

\section{Reliability}

The MBDS demonstrated very good two-week test-retest reliability. There were no differences $(p=0.13$ ) between the scores obtained at the first and second moment. The intraclass correlation coefficient (0.81) and Cronbach's $\alpha$ (moment $1=0.90$ and moment $2=0.92$ ) also indicated good test-retest reliability.

\section{Exploratory factor analysis}

The Kaiser-Meyer-Olkin measure of sampling (0.89) and Bartlett's test of sphericity $\left(\chi^{2}(300)=4367.835 ; \mathrm{p}<0.001\right)$ revealed that the data were suitable for factor analysis. In determining how many factors to retain, a scree plot was also examined, and an item analysis was conducted for various factor solutions. The scree plot suggested four possible factor solutions (Figure 1). The 2 -factor model was retained because this model provided the clearest and parsimonious item loadings. In particular, based on examination of the 3- and 4-factor solutions, it was apparent that many of the significant items loaded on several factors, or a single item was saturated by a single factor.

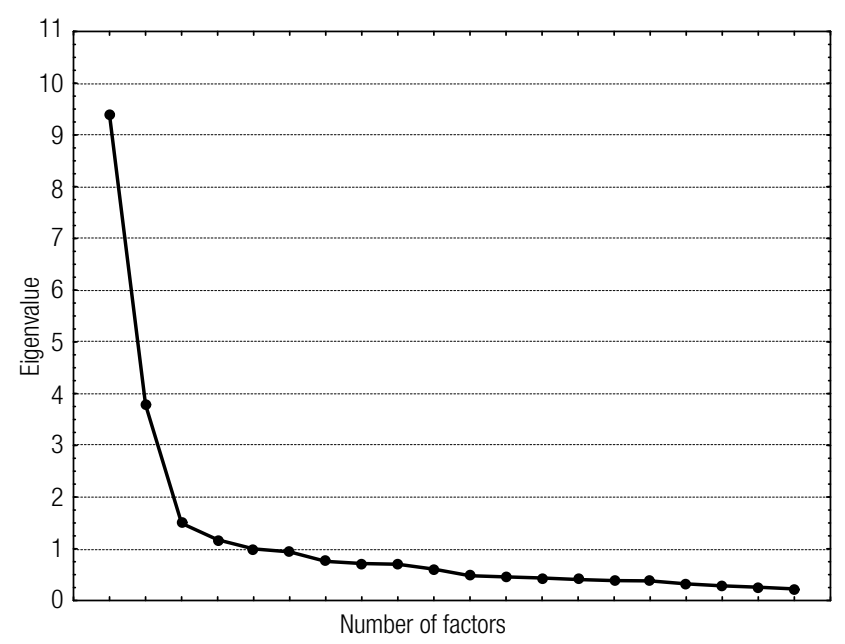

Figure 1. Scree plot of the MBDS.

The model obtained in the EFA consisted of two factors: Factor 1 ("Dissatisfaction with body muscularity"; eigenvalue $=9.38$ ) and Factor 2 ("Positive muscle attributes"; eigenvalue = 3.78). Together, these factors explained $52.67 \%$ of total variance. The internal consistency of these two factors was considered adequate (Table 2).

\section{Discussion}

The interest in the study of body image in Brazil has been increasing. However, few tools are available for assessing male body image ${ }^{2}$, and none of the available instruments allows the individual to evaluate the degree of importance of the items. The MBDS places special emphasis on addressing the potential shortcoming of other rating scales and allows each item to be weighted according to individual relevance. For these reasons, the purpose of this study was to examine the convergent validity, internal consistency, test-retest reliability and factor structure of the MBDS 19 in Brazilian men.

Regarding convergent validity, the MBDS had direct and strong associations with several measures. As an example, we can mention its relationship with drive for muscularity (DMS and SMAQ) and psychological commitment to exercise (CES). These results support the findings of Murray et al. 6 , which indicated that body dissatisfaction was strongly related to the drive for muscularity and exercise addiction/dependence.

The MBDS was also negatively associated with self-esteem (RSS) and directly associated with eating disorder risk behaviours (EAT26). These results were also observed in Murray et al.'s study ${ }^{6}$. Both, Ochner et al. ${ }^{18}$ and Rousseau et al. ${ }^{31}$ found a negative association between the MBDS and self-esteem and body self-esteem. However, Ochner et al. 18 identified a marginal association $(\mathrm{p}=0.07)$ between the MBDS and eating disorder risk behaviours (EAT-26; $r=0.32$ ).

The results indicated that the Brazilian version of the MBDS has good temporal stability with a high intraclass correlation coefficient and adequate Cronbach's $\alpha$. The principles of validity and reliability are fundamental cornerstones of the scientific method and are essential to the correct assessment of male body image and to strategies for clinical and epidemiological assessment. Similarly, the MBDS's assessment of each item's degree of importance in addition to the Likert scale responses increases the scale's reliability and avoids over- or underestimating an item's contribution to the total score. 
Table 2. The items from the Brazilian version of the Male Body Dissatisfaction Scale: factor loadings, Cronbach's $\alpha$ and explained variance obtained from the exploratory factor analysis

\begin{tabular}{|c|c|c|}
\hline Item & Factor 1 & Factor 2 \\
\hline $\begin{array}{l}\text { 1. I am happy with how much muscle I have compared to } \\
\text { how much fat I have. }\end{array}$ & & 0.73 \\
\hline 2. Other people think I have a good body. & & 0.74 \\
\hline 3. I am a good weight for my height. & & 0.54 \\
\hline 4. I wish I had more muscular arms. & 0.77 & \\
\hline $\begin{array}{l}\text { 5. I am hesitant to take my shirt off in public because } \\
\text { people will look at my body. }\end{array}$ & 0.40 & \\
\hline 6. I fantasise about having more muscle. & 0.71 & \\
\hline 7. I have thoughts of dissatisfaction with my body. & 0.73 & \\
\hline 8. I think I have a generally attractive body. & & 0.83 \\
\hline 9. I wish I had more of a V-shaped torso (upper body). & 0.52 & \\
\hline $\begin{array}{l}\text { 10. I wish I could become more toned to accentuate the } \\
\text { muscle I do have. }\end{array}$ & 0.75 & \\
\hline 11. I am more muscular than the average male my age. & & 0.72 \\
\hline 12. I worry about being more muscular. & 0.80 & \\
\hline 13. I wish I had bigger biceps. & 0.81 & \\
\hline 14. I think my pectoral (chest) muscles are well developed. & & 0.72 \\
\hline 15. I have a "six-pack" or "washboard stomach". & & 0.68 \\
\hline 16. Others would find me more attractive if I had more muscle. & 0.75 & \\
\hline 17. I wish I could lose more fat. & 0.50 & \\
\hline 18. My body looks healthy. & & 0.58 \\
\hline 19. I like to show off my body. & & 0.44 \\
\hline 20. The shape of my body is one of my assets. & & 0.69 \\
\hline $\begin{array}{l}\text { 21. I look like I could lift more weight than the average male } \\
\text { my age. }\end{array}$ & & 0.56 \\
\hline 22. I wish I had better muscle definition. & 0.77 & \\
\hline 23. My body is sexually appealing to others. & & 0.67 \\
\hline $\begin{array}{l}\text { 24. I think about how different my body looks from what my } \\
\text { ideal body would look like. }\end{array}$ & 0.79 & \\
\hline 25. I wish I could build a better body for myself. & 0.70 & \\
\hline \% explained variance. & 37.54 & 15.13 \\
\hline Cronbach's $\alpha$. & 0.90 & 0.92 \\
\hline
\end{tabular}

Factor 1: "Dissatisfaction with body muscularity"; Factor 2: "Positive muscle attributes".

With respect to the EFA, Bartlett's test of sphericity was significant, and the KMO measure was appropriate. The analysis indicated the presence of two factors named according to the items that comprised them. As previously noted, Ochner et al. ${ }^{18}$ used a reduced sample for the factor analysis; for that reason, a new EFA was performed for the Brazilian version of the MBDS. Rousseau et al. ${ }^{31}$ evaluated the factor structure of the MBDS for French adults through exploratory and confirmatory factor analysis. In the French study ${ }^{31}$, the EFA revealed two factors, "Dissatisfaction with body muscularity" (eight items: 4, 6, 9, 10, 12, 13, 16 and 22) and "General body appearance dissatisfaction" (10 items: 1, 2, 3, 5, 7, 8, 15, 17, 19 and 23 ), which explained $42.7 \%$ of the total variance of the MBDS. Seven items were excluded because they systematically glutted on several factors (4) and because they were identified before the breaking point of $0.40(3)^{31}$.

Although some items from the French version loaded to factors similar to those on the Brazilian version of the MBDS, our study's solution (two factors) fit the data better than the alternative solutions (3-4 factors). Therefore, the naming of the factors that Rousseau et al. ${ }^{31}$ suggested was not considered appropriate. In addition, the factor structure identified in the present study kept the scale's original 25 items, which is important because it allows comparisons between groups from different countries, cultures and languages. Therefore, when possible, it is desirable to maintain compatibility between the original instrument and the version adapted for other cultures.
There are limitations to this study that are important to acknowledge. First, a convenience sample was used, a fact that compromises the generalisability of the results and does not represent cultural diversity. Second, the EAT-26 was used as a proxy for eating disorder risk behaviours to test convergent validity. Although the instrument has demonstrated good psychometric properties, it has high sensitivity and low specificity25. Future studies should try to assess the discriminant validity of the MBDS by comparing a group with eating disorders (correctly identified via clinical interview) with a group without it. Third, a doubly indirect method (BMI) was used to estimate nutritional status in undergraduate students; therefore, the results are subject to measurement errors. Lastly, we note the need to conduct a confirmatory factor analysis; however, doing so would require a sample of approximately 250 individuals ${ }^{29,30}$. A confirmatory factor analysis with a large sample is planned, and further testing with a heterogeneous sample is required to determine the applicability of the MBDS to more diverse populations. In this sense, a diverse sample including men from different university courses and a variety of other socio-demographic characteristics (race, relationship and socio-economic status) should be taken into account.

Despite these limitations, this study presents empirical results that demonstrate the good validity and reliability of the MBDS ${ }^{19}$ for evaluating male body dissatisfaction in young Brazilians, thus providing an important tool for clinical and epidemiological research.

\section{Acknowledgements}

We gratefully acknowledge the instrumental support of the Laboratório de Estudos do Corpo (LABESC). We thank the study participants and the Federal University of Juiz de Fora (UFJF) for the partnership in this study.

\section{References}

1. Cash TF, Smolak L. Body image: a handbook of science, practice, and prevention. $2^{\text {nd }}$ ed. New York: Guilford Press; 2011.

2. Carvalho PHB, Ferreira MEC. Imagem corporal em homens: instumentos avaliativos. Psic Teor Pesq. 2014;30(3):277-85.

3. Bradley LA, Rudd N, Reilly A, Freson T. A review of men's body image literature: what we know, and need to know. Int J Costume Fashion. 2014;14(1):29-45.

4. Forbes GB, Jung J, Vaamonde JD, Omar A, Paris L, Formiga NS. Body dissatisfaction and disordered eating in three cultures: Argentina, Brazil, and the U.S. Sex Roles. 2012;66(9-10):677-94.

5. Santos Silva DA, Nahas MV, de Sousa TF, Del Duca GF, Peres KG. Prevalence and associated factors with body image dissatisfaction among adults in southern Brazil: a population-based study. Body Image. 2011;8(4):427-31.

6. Murray SB, Rieger E, Hildebrandt T, Karlov L, Russell J, Boon E, et al. A comparison of eating, exercise, shape, and weight related symptomatology in males with muscle dysmorphia and anorexia nervosa. Body Image. 2012;9(2):193-200

7. Helman CG. Culture, health and illness: an introduction for health professionals. Oxford: Butterworth-Heinemann; 2014.

8. Goldenberg M. The body as capital: understanding Brazilian culture. Vibrant. 2010;7(1):220-38.

9. American Society of Plastic Surgery [internet homepage]. 2013 Plastic Surgery Statistics Report. Available from: http://www.plasticsurgery.org/. Accessed on: Nov 10, 2014.

10. Cafri G, Thompson JK. Measuring male body image: a review of the current methodology. Psychol Men Mascul. 2004;5(1):18-29.

11. Dakanalis A, Timko A, Madeddu F, Volpato C, Clerici M, Riva G, et al. Are the Male Body Dissatisfaction and Drive for Muscularity Scales reliable and valid instruments? J Health Psychol. 2015;20(1):48-59.

12. Tod D, Morrison TG, Edwards C. Evaluating validity and test-retest reliability in four drive for muscularity questionnaires. Body Image. 2012;9(3):425-8.

13. McCreary DR, Sasse DK. An exploration of the drive for muscularity in adolescent boys and girls. J Am Coll Health. 2000;48(6):297-304.

14. Morrison TG, Morrison MA, Hopkins C, Rowan ET. Muscle mania: development of a new scale examining the drive for muscularity in Canadian men. Psychol Men Mascul. 2004;5(1):30-9. 
15. Yelland C, Tiggemann M. Muscularity and the gay ideal: body dissatisfaction and disordered eating in homosexual men. Eat Behav. 2003;4(2):107-16.

16. Edwards S, Launder C. Investigating muscularity concerns in male body image: development of the Swansea Muscularity Attitudes Questionnaire. Int J Eat Disord. 2000;28(1):120-4.

17. Campana ANNB, Tavares MCGCF, Swami V, Silva D. An examination of the psychometric properties of Brazilian Portuguese translations of the Drive for Muscularity Scale, the Swansea Muscularity Attitudes Questionnaire, and the Masculine Body Ideal Distress Scale. Psychol Men Mascul. 2013;14(4):376-88.

18. Ochner CN, Gray JA, Brickner K. The development and initial validation of a new measure of male body dissatisfaction. Eat Behav. 2009;10(4):197-201.

19. Carvalho PHB, Ferreira MEC, Kotait M, Teixeira PC, Hearst N, Cordás TA, et al. Equivalências conceitual, semântica e instrumental: análises preliminares da versão em português (Brasil) da Male Body Dissatisfaction Scale (MBDS). Cad Saude Publica. 2013;29(2):403-09.

20. Rosenberg M. Society and the adolescent self-image. Princeton, NJ: Princeton University Press; 1965.

21. Hutz CS, Zanon C. Revisão da adaptação, validação e normatização da escala de autoestima de Rosenberg. Aval Psicol. 2010;10(1):41-9.

22. Beck AT, Ward CH, Mendelson M, Mock J, Erbaugh J. An inventory for measuring depression. Arch Gen Psychiatry. 1961;4:561-71.
23. Gorestein C, Andrade L. Inventário de Depressão de Beck: propriedades psicométricas da versão em português. Rev Psiquiatr Clin. 1998;25(5):245-50.

24. Garner DM, Olmsted MP, Bohr Y, Garfinkel PE. The eating attitudes test: psychometric features and clinical correlates. Psychol Med. 1982;12(4):871-8.

25. Nunes MA, Camey S, Olinto MTA, Mari JJ. The validity and 4-year testretest reliability of the Brazilian version of the Eating Attitudes Test-26. Braz J Med Biol Res. 2005;38(11):1655-62.

26. Teixeira PC, Hearst N, Matsudo SMM, Cordás TA, Conti MA. Adaptação transcultural: tradução e validação de conteúdo da versão brasileira do Commitment Exercise Scale. Rev Psiquiatr Clin. 2011;38(1):24-8.

27. Streiner DL. Starting at the beginning: an introduction to coefficient alpha and internal consistency. J Pers Assess. 2003;80(1):99-103.

28. Tabachnick BG, Fidell LS. Using multivariate statistics. $6^{\text {th }}$ ed. Boston, MA: Pearson Education; 2012.

29. Hair JF, Black WC, Babin BJ, Anderson RE, Tatham RL. Análise multivariada de dados. $6^{a}$ ed. Porto Alegre: Bookman; 2009.

30. Kahn JH. Factor analysis in counseling psychology research, training, and practice: principles, advances, and applications. Couns Psychol. 2006;34(5):684-718.

31. Rousseau A, Denieul M, Lentillon V, Valls M. French validation of the Male Body Dissatisfaction Scale in a sample of young men. J Ther Comportementale Cogn. 2014;24(3):122-9. 